\title{
Superconducting phase qubits
}

\author{
John M. Martinis
}

Published online: 18 February 2009

(C) The Author(s) 2009. This article is published with open access at Springerlink.com

\begin{abstract}
Experimental progress is reviewed for superconducting phase qubit research at the University of California, Santa Barbara. The phase qubit has a potential advantage of scalability, based on the low impedance of the device and the ability to microfabricate complex "quantum integrated circuits". Single and coupled qubit experiments, including qubits coupled to resonators, are reviewed along with a discussion of the strategy leading to these experiments. All currently known sources of qubit decoherence are summarized, including energy decay $\left(T_{1}\right)$, dephasing $\left(T_{2}\right)$, and measurement errors. A detailed description is given for our fabrication process and control electronics, which is directly scalable. With the demonstration of the basic operations needed for quantum computation, more complex algorithms are now within reach.
\end{abstract}

Keywords Quantum computation - Qubits · Superconductivity · Decoherence

PACS 03.65.Yz $\cdot 03.67 . \mathrm{Lx} \cdot 85.25 . \mathrm{Cp}$

\section{Introduction}

Superconducting qubits are a unique and interesting approach to quantum computation because they naturally allow strong coupling. Compared to other qubit implementations, they are physically large, from $\sim 1 \mu \mathrm{m}$ to $\sim 100 \mu \mathrm{m}$ in size, with interconnection topology and strength set by simple circuit wiring. Superconducting qubits have the advantage of scalability, as complex circuits can be constructed using well established integrated-circuit microfabrication technology. 

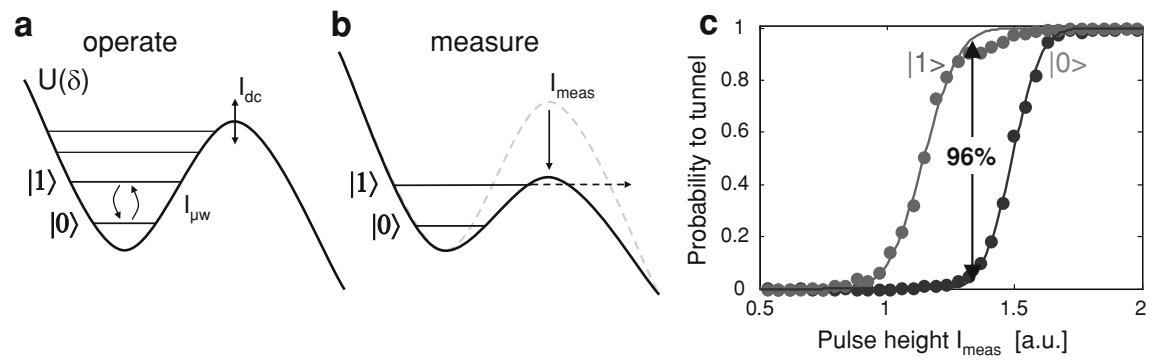

Fig. 1 a Plot of non-linear potential $U(\delta)$ for the Josephson phase qubit. The qubit states $|0\rangle$ and $|1\rangle$ are the two lowest eigenstates in the well. The junction bias $I_{\mathrm{dc}}$ is typically chosen to give 3-7 states in the well. Microwave current $I_{\mu \mathrm{w}}$ produces transitions between the qubit states. b Plot of potential during state measurement. The well barrier is lowered with a bias pulse $I_{\text {meas }}$ so that the $|1\rangle$ state can rapidly tunnel. c Plot of tunneling probability versus $I_{\text {meas }}$ for the states $|0\rangle$ and $|1\rangle$. The arrow indicates the optimal height of $I_{\text {meas }}$, which gives a fidelity of measurement close to the maximum theoretical value $96 \%$

A key component of superconducting qubits is the Josephson junction, which can be thought of as an inductor with strong non-linearity and negligible energy loss. Combined with a capacitance, coming from the tunnel junction itself or an external element, a inductor-capacitor resonator is formed that exhibits non-linearity even at the single photon level. As shown in Fig. 1, the two lowest quantum eigenstates of this non-linear resonator can then be identified as the qubit states $|0\rangle$ and $|1\rangle$.

The junction non-linearity can be expressed in circuits by different ways, leading to qubit types that have been named [6] charge, flux, transmon [19], and phase qubits. As discussed in a previous review article [6], each type has certain advantages and disadvantages. At UC Santa Barbara, we have focussed on the phase qubit because it has the largest capacitance and consequently has a characteristic resonator impedance close to $50 \Omega$. This is a convenient impedance since microwave circuits are typically designed for $50 \Omega$, and impedance matching implies that phase qubits can be strongly coupled to transmission lines and resonator circuits. Similarly, phase qubits can be directly wired together over relatively long distances, since the stray capacitance of the wires can be made negligible compared to the junction capacitance. Although impedance has not been a critical issue for present proof-of-principle experiments, we believe this circuit property may become increasingly important as more complex integrated circuits are realized.

Note that these same wires that permit scaling to large qubit architectures also allow qubits to interact with unwanted defects and electromagnetic modes, increasing the possibility for dissipation and decoherence. Understanding decoherence is thus a critical issue for all superconducting qubits, and will be specifically addressed in this review.

This article is not intended to be a comprehensive explanation of phase qubits, but more a guide to publications by the Santa Barbara group in the last few years. I will mostly review the strategy, concepts, and implications of these experiments. In addition, some important details have been left out of prior publications, notably fabrication and control electronics, and this review will be used to more fully document these issues. Since many of the experiments demonstrated advances in several 
areas, the review will be structured along topics, listing chronologically the important developments when appropriate.

\section{Single qubits}

Superconducting qubit research began in the 1980s motivated by the question, posed by Anthony Leggett, whether macroscopic variables would behave in a quantum mechanical fashion [23]. Initial experiments verified quantum behavior via the phenomenon of tunneling out of the zero-voltage state of a current-biased Josephson junction [7]. At UC Berkeley, quantum mechanical behavior was also demonstrated by the existence of quantized energy levels [28]. This observation provided stronger proof of quantum behavior, and established at an early stage (before the ideas of qubits were even widely established) that superconducting circuits could be used as general quantum systems [3].

Motivated by theoretical developments in quantum computing, research into phase qubits was renewed in the early 2000s. The University of Maryland group proposed in 2001 that qubits could be encoded as the ground and first excited state of a current-biased Josephson junction [39]. The phase qubit was first demonstrated at NIST in 2002 with an experiment [30] that performed all of the basic operations needed for a single qubit: initialization of the qubit state, coherent Rabi oscillations, and a probabilistic single-shot measurement of the final state. An important conceptual advance in this circuit was biasing the junction with a magnetic flux applied to superconducting loop, which provided a large impedance transformation that isolated the qubit from the strongly dissipative $50 \Omega$ impedance of the bias leads. Another advance was measurement, which was performed by first driving a transition from $|1\rangle$ to a higher energy eigenstate, which would then rapidly tunnel out of the well to produce an easily measured voltage signal.

This initial experiment obtained a coherence time of approximately $20 \mathrm{~ns}$, a value much smaller than $\sim 4 \mu$ s expected from damping of the bias leads. To probe the cause of this discrepancy, the fabrication procedure was first changed, replacing the $\mathrm{Nb}$ superconductor with $\mathrm{Al}$, since long $\sim 1 \mu$ s coherence times had been already been achieved [52] with charge qubits using Al. This change in materials did not improve the qubit coherence, so we next investigated the role of quasiparticles that were generated in the junction when switching into the voltage state [22]. Because the qubit escape rate was found to be influenced by changes to the generation and decay of quasiparticles, the qubit design was more radically modified so that quasiparticles would not be produced by the qubit junction during measurement [45].

All subsequent qubits are based on this new design, which is shown in Fig. 2 for our current layout. The qubit junction is still biased through a superconducing loop, but now the tunneling event simply changes the circulating current in the loop. This current change is read out with a separate SQUID magnetometer that is connected only by flux, electrically isolating the qubit junction from the readout so that any quasiparticles generated in the readout cannot diffuse into the qubit junction. In addition, an external shunt resistor is also connected across the SQUID so that it switches to about $1 / 4$ th the gap voltage [22]. This minimizes the generation of quasiparticles, and 

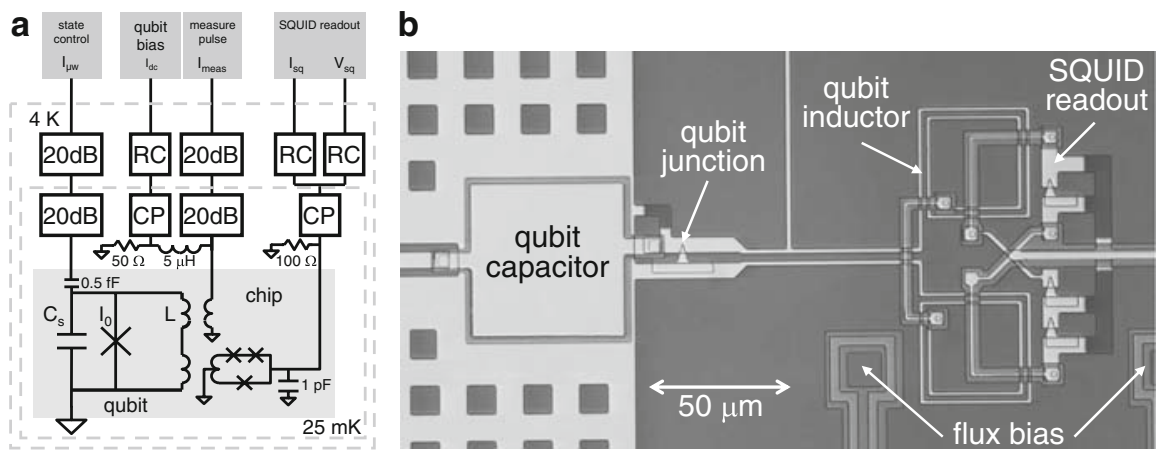

Fig. 2 a Schematic diagram of a phase qubit circuit and bias lines. Symbols $20 \mathrm{~dB}$, RC, and CP represent $20 \mathrm{~dB}$ microwave attenuators, resistor-capacitor low-pass filters, and copper-powder microwave filters, respectively. The RC filters for the qubit and SQUID bias has $1 \mathrm{k} \Omega$ and $10 \mathrm{k} \Omega \mathrm{DC}$ resistance, respectively, and a roll-off frequency $\sim 5 \mathrm{MHz}$. The $5 \mu \mathrm{H}$ inductor is a custom made radio-frequency bias tee with no transmitting resonances below $1 \mathrm{GHz}$. b Photomicrograph of present phase qubit, showing small area $\left(\sim 1 \mu \mathrm{m}^{2}\right)$ junction shunted by a parallel plate capacitor. Microwave drive line (with capacitor, not shown) comes from the left. The qubit inductor is coupled to a SQUID readout circuit in a gradiometer geometry. The flux bias lines for the qubit are symmetrically placed about the SQUID and counter-wound to inhibit flux coupling to the SQUID. The SQUID bias line exits to the right. The holes in the ground plane inhibit trapped vortices in the superconducting ground plane

ensures that the Josephson oscillations have a frequency smaller than the gap so that no quasiparticles can be excited in the qubit loop. Using this new design, we have not seen any effects of quasiparticle heating, validating the design change. Also note that a gradiometer design is currently used for both the qubit and readout SQUID to minimize flux offsets from trapped magnetic flux in the ground plane.

With this new design, the qubit coherence time was unchanged. Careful spectroscopy sweeps in bias were performed for the first time, which showed structures corresponding to avoided level crossings [45]. These "spurious resonances" were shown to be consistent with a model of the qubit interacting with two-level states (TLS) produced by atoms in the dielectric tunneling between two sites [38] (or more generally the tunneling between two configurations of atoms.) Although these resonances were initially hypothesized to fluctuate the critical current, it was later proposed [26] and demonstrated [27] that they instead produce a fluctuation in charge.

In the next publication [4], we improved state measurement by applying a $\sim 3 \mathrm{~ns}$ pulse to the qubit bias line, causing the $|1\rangle$ state to directly tunnel, as illustrated in Fig. 1b. With faster measurement, we were then able to show that the qubit $|1\rangle$ state, when biased on resonance with the two-level fluctuator, could Rabi swap the excited photon. The magnitudes and density of these TLS fluctuators were also shown to explain why the measurement probabilities of Rabi oscillations were about $1 / 2$ the expected magnitude.

The breakthrough in phase qubit coherence came in 2005 [27], when we first identified the source of the spurious resonators to be TLS in the dielectrics (insulators) of the device. With conventional inductor-capacitor resonators, we first showed that a common dielectric used in superconducting devices, amorphous a-SiO intrinsic dielectric loss tangent $\delta_{i} \simeq 0.005$. This loss was shown to decrease at high 
power or temperature because of saturation effects. We then showed that the spurious resonances have a distribution of splitting sizes, as measured with qubit spectroscopy, consistent with individual TLS in the $\mathrm{AlO}_{x}$ dielectric of the tunnel junction. The density of splittings were calculated to give $\mathrm{AlO}_{x}$ an intrinsic loss tangent 0.0016 , close to the $\mathrm{a}-\mathrm{SiO}_{2}$ value. We also demonstrated that the splitting density increases with junction area, and argued that large area junctions $\left(A>100 \mu \mathrm{m}^{2}\right)$ have enough overlapping splittings so that this loss tangent directly determines the energy loss time. This calculated time $T_{1}=8 \mathrm{~ns}$ correctly predicts what was found in previous experiments on large area qubits. For small area junctions $\left(A<1 \mu \mathrm{m}^{2}\right)$, much longer coherence times are observed because the qubit bias can be chosen to statistically avoid the splittings. Charge and flux qubits must use small area junctions, which explains why this phenomenon was not originally seen in those devices. This paper also reported that the loss tangent of a-SiN $x$ is about 20 times better than $\mathrm{a}-\mathrm{SiO}_{2}$. Replacing the crossover

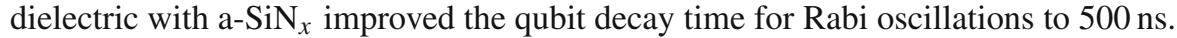

We note that this dramatic increase in $T_{1}$ came from a number of device improvements. Most importantly, the junction area was reduced and low-loss dielectrics were used in the wiring crossovers. We also switched substrates from silicon to sapphire, since we found that resonators made on silicon with CVD-deposited a- $\mathrm{SiO}_{2}$ and a-SiN $x$ dielectrics had anomalously high dissipation. This presumably comes from a conducting 2-dimensional electron gas layer at the surface of the silicon, induced by a large electric field generated by charge defects in the amorphous dielectric. We have not checked whether the redesign of the qubit to reduce quasiparticle heating, or the use of $\mathrm{Al}$ instead of $\mathrm{Nb}$, were ultimately important changes.

Although $T_{1}$ had improved, the amplitude of the Rabi oscillations was at this time only about $65 \%$. Theory predicted that this measurement fidelity could be improved by using a qubit junction with smaller area, reducing the number of TLS resonances that were swept through during the measurement pulse [4]. A device was then tested [49] that incorporated small area junctions with $A=1 \mu \mathrm{m}^{2}$ and shunted with an external capacitor in order to keep the qubit frequency constant. Spectroscopy data from this device showed a dramatic decrease in the number of splittings, with the measurement visibility jumping to about $85 \%$, as expected. The decay time decreased in this device to $T_{1}=110 \mathrm{~ns}$, consistent with the loss tangent of the $\operatorname{SiN}_{x}$ shunting capacitor. It is important to note that even though $T_{1}$ decreased in this device, the reduction in the number of TLS splittings made the qubit much easier to precisely control. We were thus able to perform for the first time a number of important experiments, including partial measurement [17], quantum-state tomography on single [49] and coupled [48] qubits, and demonstration of entanglement between two qubits [48].

The coherence time $T_{1}$ was next improved [35] by replacing the dielectric of the shunt capacitor with hydrogenated amorphous silicon $(\mathrm{a}-\mathrm{Si}: \mathrm{H})$, which we had shown in resonator experiments to have about 8 times lower intrinsic loss than a-SiN $\mathrm{N}_{x}$. Various chemical interactions of a-Si:H with $\mathrm{Al}$ makes this material much more difficult to incorporate into a multi-level process. Initial experiments $[24,34,35]$ gave $T_{1}=450 \mathrm{~ns}$, whereas more recent devices have shown decay times as long as $600 \mathrm{~ns}$. Experiments are currently underway to optimize the growth of this a-Si:H dielectric.

The best performance of a single qubit is shown in Fig. 3 for Rabi oscillations. Ramsey fringes (not shown) have given dephasing times as long as $200 \mathrm{~ns}$, although 


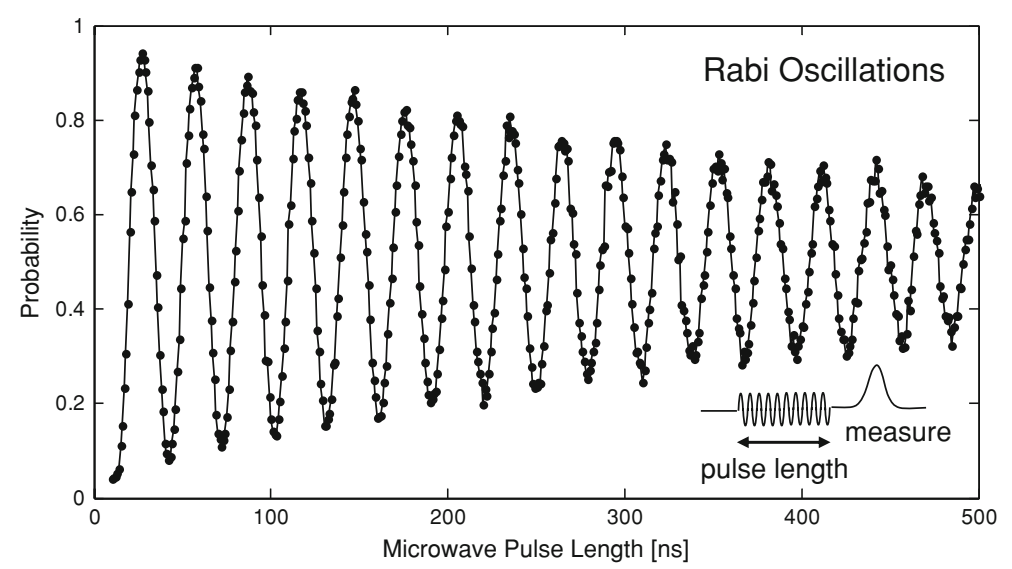

Fig. 3 Measurement of Rabi oscillations. The measurement probability of the $|1\rangle$ state is plotted versus microwave pulse length. Pulse sequence consists of a microwave pulse of variable time, tuned to the qubit transition frequency, followed by a measurement pulse, as depicted in the inset. Note that the probability is the raw data (with no corrections) coming from our experiment, which inherently has an absolute calibration. The Rabi oscillations have fidelity of about $90 \%$, a value reasonably close to the theoretical expectation $96 \%$ [4]. The energy decay time for this qubit is $T_{1}=600 \mathrm{~ns}$

we typically find $T_{2}=120 \mathrm{~ns}$ for present qubits. A spin-echo sequence [29] has been shown to increase the dephasing time to about $300 \mathrm{~ns}$.

At this time, a new generation of electronics gave microwave phase control [17], allowing the qubit state to be rotated around any axis on the equator of the Bloch sphere. With microwaves applied slightly out of resonance, rotation off-axis from the equator also became possible. We also improved the flux bias line by DC coupling the measurement pulse and installing a bias-tee inductor to the (low frequency) DC bias line. These improvements allowed fast and accurate current pulses to be applied to the qubit, enabling precise control in time of the qubit frequency, and giving phase control of the qubit state through rotation around the pole (z-axis) of the Bloch sphere. Experiments on single qubit tomography demonstrated this Z-gate control [49].

\section{Partial measurement}

The phase qubit is typically measured through tunneling of the $|1\rangle$ state. Because the tunneling rate can be electrically adjusted, it is possible to vary the probability for tunneling, and thus the measurement probability. Such partial measurements [17] allows one to "look inside" the phenomenon of state collapse in order to understand the evolution of a quantum state due to the measurement process.

In an ideal quantum measurement, the qubit state is determined without error. The eigenstates $|0\rangle$ or $|1\rangle$ are measured always as 0 or 1 , respectively. For a superposition state such as $(|0\rangle+|1\rangle) / \sqrt{2}$, the measurement probability becomes 0.5 .

Errors of a few percent arise in our present method of state measurement [4,24] because the ratio of the tunneling rates from the $|0\rangle$ and $|1\rangle$ states is finite, typically $\sim 200$. The measurement probabilities from these errors, along with small errors from 
the qubit state relaxing to the TLS during the measurement pulse [4], can be corrected for using calibrations and simple linear algebra [48].

The information available from the result of a partial measurement also produces a change to the quantum state. For perfect measurement, the qubit state is projected into a known state. For partial measurement, the state still undergoes coherent evolution that can be experimentally checked. A good simplification for our system is to assume that the $|0\rangle$ state does not tunnel, but that the $|1\rangle$ state can tunnel (be measured) with a probability $p$ that can be adjusted with the measurement bias pulse, as shown in Fig. 1c. An initial quantum state $\psi_{i}$ then probabilistically evolves to two final states, with states and probabilities given by

$$
\psi_{i}=a|0\rangle+b|1\rangle \rightarrow\left\{\begin{array}{ccc}
\psi_{t} & p_{1} & \text { (tunneled) } \\
\psi_{f}=(a|0\rangle+b \sqrt{1-p}|1\rangle) / \sqrt{N} & 1-p_{1} & \text { (not tunneled) }
\end{array}\right.
$$

where the initial state is normalized $|a|^{2}+|b|^{2}=1$, the probability for tunneling is $p_{1}=|b|^{2} p$, the state of the system after tunneling is $\psi_{t}$, and $N=|a|^{2}+(1-p)|b|^{2}$ is the normalization of the final state $\psi_{f}$ when not tunneling. For the case of perfect measurement $(p=1)$ the final state collapses to $|0\rangle$ when not tunneled, as expected. For partial measurement, the final state is in a superposition of $|0\rangle$ and $|1\rangle$, and has changed by a coherent but non-unitary evolution from $\psi_{i}$ to $\psi_{f}$. This state evolution was verified with a phase qubit, with the final state measured with state tomography [17].

The non-unitary evolution can also be undone or "uncollapsed" using a second identical measurement pulse, after first performing a $\pi$-rotation to exchange the $|0\rangle$ and $|1\rangle$ state. This operation can be considered as a generalization of spin-echo to non-unitary operations. An experiment showed that that the original state is accurately restored after these two partial measurements [18].

\section{Coupled qubits}

Qubits need to be coupled together to produce entangled states and to perform complex quantum logic operations. The coupling of two qubits, together with single qubit operations, allow generation of the CNOT gate, a fundamental operation from which more complex transformations can be constructed.

Phase qubits have been coupled via a capacitance that allows a photonic excitation to pass between two qubits. This interaction Hamiltonian is of the form

$$
H_{\text {int }}=g(|10\rangle\langle 01|+| 01\rangle\langle 10|)
$$

with a coupling strength $g$ that is proportional to the coupling capacitance. If $H_{\text {int }}$ is turned on for at time $t$ such that $g t=\pi$, the initial state $|01\rangle$ evolves to the state $-\mathrm{i}|10\rangle$; the swapping of the photon along with the $-\mathrm{i}$ phase factor suggests naming this operation i-SWAP.

An initial experiment on capacitively coupled phase qubits [1] focused on the spectroscopy of qubits, demonstrating an avoided level crossing with an energy splitting of 
$2 g$. Experiments by the NIST and UCSB group focussed on testing the time dynamics of this coupling, as needed for understanding how to make a two-qubit gate. All experiments to date have used fixed capacitive coupling. Single qubit operations were performed by having them operate on a time scale much faster than the coupling interaction. Later experiments have effectively turned on and off this coupling by biasing the qubits into and out of resonance.

Our first experiment demonstrated swapping of a photon between a qubit and a two-level state [4]. Here, the swapping operation was inferred by measuring the oscillating probability (in time) of only the qubit. In a second publication [33], we performed this swapping experiment between two phase qubits, simultaneously measuring the states of both qubits. Simultaneous measurement was a significant advance, since it allows a unambiguous determination of the time dynamics of the two coupled qubits. It is challenging to perform, since the capacitive coupling is always on and thus couples the qubits during measurement. This causes the measurement of the $|1\rangle$ state in one qubit to sometimes excite the tunneling of the $|0\rangle$ state in the other qubit, producing measurement error. Guided by simple theory and numerical simulations, we were able to demonstrate that "measurement cross-talk" could be dramatically reduced by overlapping the timing of the two measurement pulses. Further theoretical work was able to accurately predict the tradeoff between coupling and crosstalk [21].

After improving the coherence time of phase qubits, we were able to more accurately measure the state transformation for this swapping operation [48]. We first measured the i phase factor of the i-SWAP gate by exciting one of the qubits, creating the $|01\rangle$ state, and then allowing the qubits to couple for a time $t=\pi / 2 g$, generating the entangled state $|01\rangle-\mathrm{i}|10\rangle) / \sqrt{2}$. After then applying a $\pi / 2$ phase rotation via a Z-gate to one of the qubits and removing the i phase factor, we obtained an entangled eigenstate. We confirmed that measurements of both qubit states did not oscillate in time. A full determination of the entangled state was performed with tomography, which required applying microwave pulses to each qubit before measurement to change the measurement basis. The density matrix for the entangled state was found to have off diagonal elements with an i phase factor, as expected, and with a magnitude nearly that of the diagonal elements. The fidelity of the density matrix was high enough to definitively show that the state was entangled.

This experiment with state tomography showed correct state transformation starting with one (important) initial state. In order to demonstrate that the coupling operation works properly for any initial state, state tomography must be performed over a number of initial states [34]. This process tomography experiment is currently underway at UCSB.

Since the CNOT gate can be constructed from two $\sqrt{\mathrm{i}-\mathrm{SWAP}}$ operations, measuring the fidelity of $\sqrt{\mathrm{i}-\mathrm{SWAP}}$ is crucial. We have found that the oscillation amplitude of i-SWAPs is a powerful method to determine the quality of the coupling operation, much like the use of Rabi oscillations to determine the quality of single-qubit operations. In Fig. 4 we show recent results for our coupled qubits, which demonstrate higher amplitude and longer coherence than given in previous publications. 


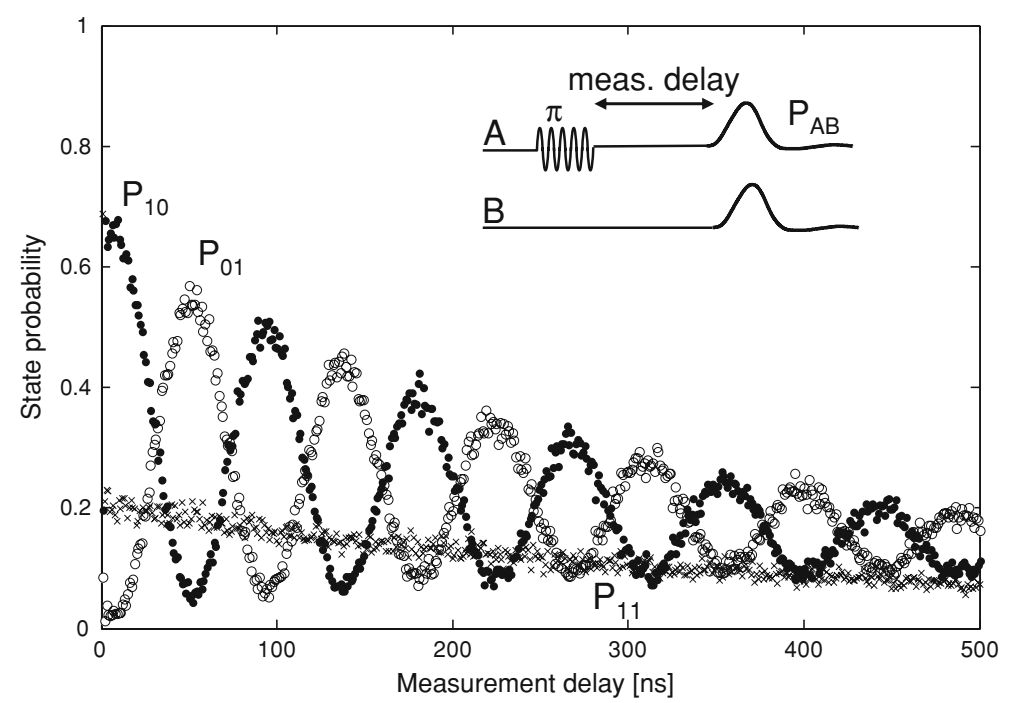

Fig. 4 Plot of Rabi swapping between the two-qubit states $|10\rangle$ and $|01\rangle$, which produces an entangling operation that leads to a CNOT gate. The probability for measuring the $|10\rangle,|01\rangle$, and $|11\rangle$ states are given by the closed circles, open circles, and $\times$ symbols, respectively. The experimental sequence is first a short $\pi$ pulse to excite $|10\rangle$, followed by a delay time where the states interact and swap $|10\rangle \leftrightarrow|01\rangle$, and then a final simultaneous measurement of both qubit states, as depicted in the inset. The probability for $|11\rangle$ arises from a crosstalk error in measurement, where the tunneling of the $|1\rangle$ state in one qubit excites the tunneling of the $|0\rangle$ state in the other qubit with a probability $\sim 20 \%$. Adding the $20 \%$ probability to $P_{10}$ yields a visibility of about $90 \%$, which corresponds to the full measured visibility of single qubits, as seen in Rabi oscillations. We thus expect large visibility for the swapping operation once all measurement errors are taken into account

\section{Qubit-resonator experiments}

Superconducting qubits are electrical resonators with a non-linearity that is strong enough so that microwaves excite transitions only between the $|0\rangle$ and $|1\rangle$ states. Resonators, built from inductors and capacitors or from standing waves in transmission lines, are linear oscillators that have eigenstates equally spaced in energy. Transitions are on-resonance between every adjacent pair of eignestates, yielding dynamical behavior much different than for a qubit.

Resonators can be driven or probed with large amplitude signals, and lead to a number of interesting physical effects and possible applications such as qubit readout. Resonators are also easier to fabricate than qubits because they do not require Josephson junctions, potentially giving a lower loss circuit that can be used for quantum memory [34]. The large size of a transmission line resonator may enable their use as a quantum bus to couple qubits over long distances $[25,44]$.

A phase qubit coupled to a resonator was first demonstrated in the late 1980s, when spectroscopy experiments at Saclay showed how the qubit energy levels and their dissipation rates were modified by the complex impedance of a damped transmission line [5]. By varying the length of the transmission line, the characteristic time of tunneling was also measured [51]. These experiments were the first to demonstrate that 
any superconducting qubit could be strongly coupled to a resonator. This is easier to accomplish, compared to other superconducting qubits, because the characteristic impedance of phase qubits $(\sim 10 \Omega-100 \Omega)$ is similar to that of transmission line resonators $(50 \Omega)$. In the past few years, charge and transmon qubits coupled to resonators have demonstrated the "strong-coupling" limit [53].

As for coupled qubits, our experimental strategy has been to measure the dynamics of the coupling in the time domain. Our first experiment [15] used a device that capacitively coupled a qubit to a half-wavelength transmission line resonator. By repeatedly exciting the qubit to the $|1\rangle$ state and transferring the photon into the resonator, Fock states were sequentially generated in the resonator. This state was then analyzed by swapping these photons back and forth between the resonator and qubit, with the swap frequency being shown to be proportional to the square-root of the photon number. In this initial experiment, Fock states with up to 6 photons were created and analyzed.

A second experiment [54] yielded a resonator with a factor of 3 improvement in the energy decay, yielding a $T_{1}$ for the resonator of $3 \mu \mathrm{s}$. We analyzed the decay rate of the Fock states and demonstrated the rates scale as the photon number for Fock states from 1 to 5. The improved coherence time also increased the fidelity of the Fock states and allowed them to be generated up to photon number 15 .

The latest device we have measured has $T_{1}=5 \mu \mathrm{s}$. By generalizing the sequence of operations sent to the qubit, we have been able to create arbitrary photon states up to 9 photons [14]. The state now can be fully measured, including phase information of each Fock state, using Wigner tomography.

These resonator experiments also demonstrate precise quantum control of superconducting qubits. They have required the most complex sequences to date, as the production and detection of a 15-photon Fock state uses 32 pulses, all of which have to be properly designed, calibrated, and electronically generated.

\section{Coherence}

Understanding coherence in superconducting qubits is more than reporting the best values of $T_{1}$ and $T_{2}$ that have been achieved. This section will focus on the physical understanding of decoherence mechanisms, as discovered and explored in a number of experiments on phase qubits. A systematic picture of decoherence will be presented that hopefully will be a guide to improving the materials and design of future qubits.

A qubit can be understood as a non-linear resonator made from an inductor and capacitor [6], as illustrated in Fig. 5. Non-linear inductors are made from Josephson junctions, and linear inductors from superconducting wires. The capacitor often comes from the Josephson junction, formed by the overlapping superconducting electrodes across the thin dielectric of the tunnel barrier, typically a- $\mathrm{AlO}_{x}$. Capacitance may also come from an external element placed across the junction, deliberately designed into the device. Additionally, the qubit is also coupled to external electromagnetic modes, either from various bias lines that are used to control or measure the qubit state, or inadvertently created, for example, by radiation. Coupling to these external modes is typically considered as a mechanism for dissipation and energy loss. These three types of elements form a natural classification of possible decoherence mechanisms. 


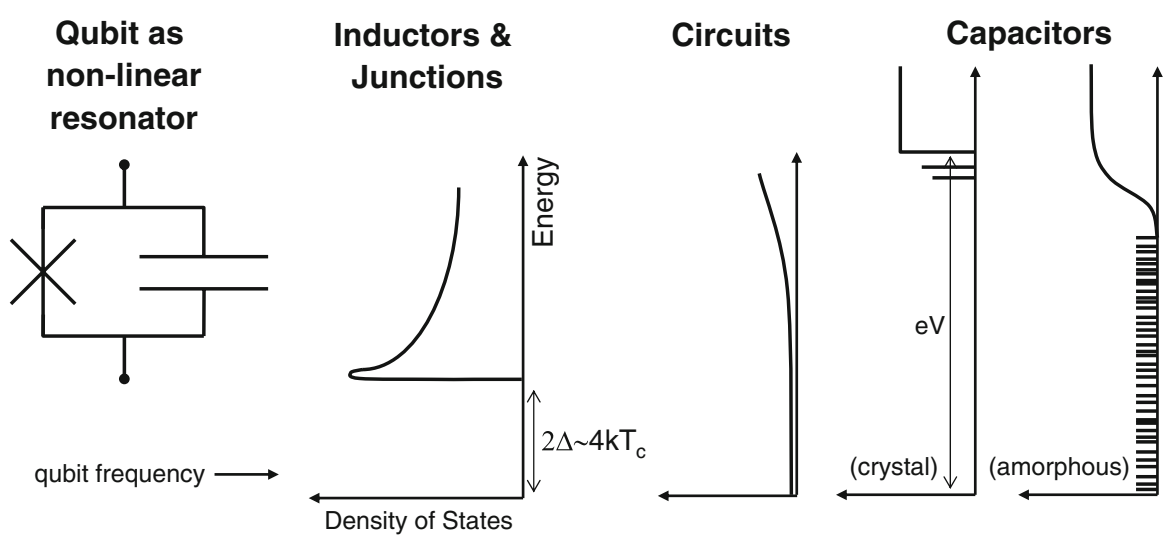

Fig. 5 General schematic representation of a Josephson qubit as a non-linear inductor-capacitor resonator. The inductive element of the resonator, coming from Josephson junctions and physical inductors, has dissipation governed by the density of superconducting states. As drawn in the plot of density of states versus energy, the superconducting gap gives no states for energies less than twice the superconducting gap $\Delta$. Dissipation coming from the leads of the resonator are represented by external circuits, and are designed to have low density of states at microwaves frequencies for low dissipation. Capacitors made from crystalline materials also have a gap in the density of states, with gap energies for typical insulators in the electron-volt range. However, amorphous insulators typically have an significant density of TLS throughout this gap region

Energy loss from the inductive elements, including the Josephson junction, is expected to be very low because the energy gap of the superconducting state provides no states to which energy can dissipate. Niobium cavity resonators [12], for example, have been measured to have quality factors $Q \sim 10^{10}$. Qubits are typically made using aluminum, which has a transition temperature $T_{c} \simeq 1 \mathrm{~K}$ and an energy gap of about $80 \mathrm{GHz}$. Because the qubit has a frequency typically in the 5 to $20 \mathrm{GHz}$ range and operates at very low temperatures $T<T_{c} / 20$, no dissipation is expected. An important property of superconductors is that the gap is retained even under the presence of disorder-perfectly so for energy conserving scattering, and with only a small smearing of the gap for energy loss mechanisms, such as arising from dilute concentrations of magnetic defects. Even the Josephson effect has a gap protected from such imperfections [37]. Its non-linear inductance is fundamentally created by Andreev bound states across the junction. For tunnel junctions with small tunneling matrix elements, these states retain a gap nearly that of the bulk value. Even for an imperfect junction with microshorts, giving some conduction channels with large transmission, the Andreev bound states retain a large energy gap for the typical operating bias of a phase qubit [37].

Although it is often stated that low loss in superconductors is an inherent advantage of these qubits, this is only half the story. Resonators are made from inductors and capacitors, and energy loss from capacitors is clearly equally important. Unfortunately, nature is not so kind concerning dielectrics. Although crystalline dielectrics have very small loss tangents, probably below $10^{-6}$, the amorphous dielectrics that are commonly used in conventional superconducting devices have large loss. For example, dielectric loss tangents of $\delta \sim 10^{-3}$ are found for the amorphous aluminum 
oxide in the tunnel junction and amorphous silicon dioxide a-SiO 2 used for insulating layers [27,36]. The important difference between dielectrics and superconductors is the physics of defects: they significantly affect dielectrics, but not the many-body superconducting state.

The bias and control circuit that is connected to a superconducting qubit produces both energy loss and fluctuations of the bias value. This latter noise generates dephasing of the qubit state. Both of these decoherence mechanisms will be discussed in the following subsections.

\section{$6.1 T_{1}$ : energy decay}

Energy loss in the qubit causes the $|1\rangle$ state to decay to the $|0\rangle$ state at a rate typically described by an inverse decay time $1 / T_{1}$. A description of several known energy loss mechanisms is reviewed here.

The best understood energy decay mechanism comes from radiation of the qubit energy to the leads attached to the device, often called the environment. The quantum mechanical treatment of this energy loss mechanism is equivalent to the radiation of light from an atom. A general calculation for the energy loss rate was first performed in 1980s [8], where it was found that the decay rate was proportional to the real part of the admittance shunting the junction, evaluated at the qubit transition frequency. The important physical insight here is that the effect of the environment is described by the admittance, which can be calculated as a classical current-to-voltage response function of the leads using conventional circuit analysis. This work also showed that the quantum decay rate is well approximated by the classical loss rate seen by a linear oscillator [8]. Energy loss can thus be understood and predicted using well-known classical concepts. This theory was experimentally tested in the late 1980 s with a variable admittance that was adjusted in-situ by changing the length of a transmission line [5].

Although the admittance function gives the experimentalist a well defined procedure for calculating loss, we found that additional insight was needed to imagine how circuits should be constructed in the first place. To do this, the concepts of impedance and current transformation were shown to be closely related, which provided both a qualitative and quantitative understanding how to isolate the qubit from the environment [35]. This concept of current transformation was accurately tested using a variable coupling circuit made from our measurement SQUID [35]. In this work we also calculated higher order non-linear effects due to mixing.

Although we have a fundamental theory for calculating energy loss, this theory depends on properly modeling the circuit. Further progress will probably entail understanding other possible energy loss mechanisms and how to model them. In particular, what radiation effects are coming from the finite size of the circuit components? A ground plane is used in phase qubit circuits to tie together a common electrode for all qubits-How does the ground plane modify radiation? Preliminary experiments suggest that these issues will be important for qubits with $T_{1}>500 \mathrm{~ns}$ and for resonators with quality factors $Q>10^{5}$.

We have also investigated an energy loss mechanism coming from trapped vortices in the superconducting ground plane [47]. For coplanar transmission line resonators, 
we have measured a reduction of the quality factors in the $10^{4}$ to $10^{5}$ range arising from vortices mainly in the center conductor [32]. The loss depends on the geometry of the transmission line and materials, and rises with increasing vortex density. Energy loss comes from motion of the vortex or current flowing through the normal metal core. This loss mechanism can be avoided by fabricated holes in the superconducing ground plane, as trapping flux in a hole does not have a normal metal core that can dissipate energy. We cool qubit devices in a mu-metal shield so that the magnetic field is low enough to ensure that all the field can be accommodated by vortices in the holes [47].

One of the most important energy loss mechanisms is dissipation from TLS in dielectrics [38], as shown in a number of our publications. As discussed previously in Sec. 2, an understanding of this loss mechanism was key to improving phase qubits. Important facts and issues are summarized here.

\section{General TLS model}

1. Dielectric loss from TLS is large for common amorphous materials [38], and thus is critical to consider for qubit designs. Dielectric loss is potentially important even from the thin surface oxide of superconducting metals [11].

2. TLS are known to arise in amorphous materials from the random bonding of atoms [38].

3. The effects of TLS are seen in both phonon and electrical characteristics [38].

4. TLS are modeled as atoms tunneling between two positions or atomic configurations. The physical size of this fluctuation is consistent with our experimental measurements of the maximum splittings seen in qubit spectroscopy [27].

5. The exponential dependence of the tunneling rate on parameters give a log-normal distribution of tunneling and TLS energies. We have verified this distribution for TLS in tunnel junctions [27].

6. The log-normal distribution is related to $1 / f$ noise [43].

7. The energy decay time of TLS [34] is estimated to be in the $10 \mathrm{~ns}$ to $1 \mu$ s range.

8. We have occasionally observed TLS to appear and disappear in time, consistent with a model where TLS are coupled to one another.

9. The observed TLS spectrum changes when a device is heated up between $4 \mathrm{~K}$ and $77 \mathrm{~K}$, presumably from annealing of the TLS atomic bonds.

10. In a charge qubit, the bias dependence of the electric field in the junction has been observed to change the TLS frequency.

11. TLS are thought to be responsible for phase noise seen in microwave coplanar resonators [10].

\section{Materials properties}

12. The loss tangent of crystalline materials such as silicon and sapphire is very small, probably below $10^{-6}$.

13. The loss tangent of amorphous oxide materials, such as a- $\mathrm{AlO}_{x}$ and $\mathrm{a}-\mathrm{SiO}_{x}$, is in the range $2 \cdot 10^{-3}$ [27].

14. The loss tangent of $\mathrm{a}-\mathrm{SiO}_{x}$ scales with the impurity concentration of $\mathrm{OH}$ [41].

15. a-SiN $x$ has 5-10 times lower loss than $\mathrm{a}_{-} \mathrm{SiO}_{x}$ [27]. 
16. a-Si:H has 5-10 times lower loss than a-SiN ${ }_{x}$ [36].

17. The lower loss of a-SiN $\mathrm{N}_{x}$ and a-Si:H is believed to arise from the greater coordination (number of bonds) of the $\mathrm{N}$ and $\mathrm{Si}$ atoms, which more tightly constrains the position of the atoms [38].

18. Dielectrics grown with high stress may give lower dielectric loss because of a greater average bond number. The optimal stress of amorphous materials is probably compressive.

19. A summary of known dielectric loss tangents from TLS is tabulated in Ref. [36].

\section{Measuring dielectric loss}

20. TLS saturate at high power or temperature, removing its dissipative effect [27].

21. Measurement of the intrinsic loss tangent $\delta_{i}$ from TLS, appropriate for qubits, must be done at low temperature and power.

22. Errors in measurement, such as increased temperature or power, tend to give a lower value of $\delta_{i}$.

23. The non-linearity of TLS dissipation can cause instabilities when measuring a resonator quality factor. For example, the lowering of the $\mathrm{Q}$ with a decrease in excitation power can cause the resonance response to rapidly disappear.

24. TLS also contribute a capacitive term, which is temperature (and weakly power) dependent [11]. The change of the resonance frequency with temperature can be used to determine $\delta_{i}$.

\section{TLS in Josephson junctions}

25. The number of TLS defects in a tunnel junction is small because the oxide is thin $(\sim 1.5 \mathrm{~nm})$ [27].

26. TLS defects can be statistically avoided using small area junctions $A<1 \mu \mathrm{m}^{2}$ [27].

27. Junctions with area $A>100 \mu \mathrm{m}^{2}$ see effectively a continuum of TLS, with $T_{1}$ predicted by the loss tangent of a-AlO $\mathrm{A}_{x}$ [27].

28. TLS lower measurement fidelity of the $|1\rangle$ state by removing energy from the qubit [4]. It is more difficult to statistically avoid the effects of TLS during measurement because the qubit is often swept through a large frequency range.

29. The loss tangent for $\mathrm{a}-\mathrm{AlO}_{x}$ in tunnel junctions is similar to its bulk value $[27,36]$. This implies that interface effects are small even for a thin $(\sim 1.5 \mathrm{~nm})$ tunnel junction dielectric, as expected since TLS are defects with an atomic size.

30. TLS have also been observed in charge and flux qubits, with a density (per junction area) compatible with that measured in phase qubits.

31. We have fabricated qubits with amorphous AlN tunnel junctions. The density of TLS was found to be approximately the same as for $\mathrm{AlO}_{x}$ barriers. In this qubit we measured a short energy decay time $T_{1} \sim 10 \mathrm{~ns}$, a value compatible with loss from phonon radiation due to AlN being piezoelectric [16].

Currently, the best decay time we have obtained for a phase qubit is $T_{1}=600 \mathrm{~ns}$. Decay times in the range of 400-600 ns are consistently found in all qubits we test. 
For resonators, although the best energy decay time is $5 \mu \mathrm{s}$, we have found times in the $1-5 \mu$ s range depending on the layout of the resonator. The shorter $T_{1}$ of the qubit is probably limited by either dielectric loss of the a-Si:H in the shunt capacitor, radiation effects, or some unknown loss mechanism in the Josephson junction.

Significant improvements in $T_{1}$ can probably be made by using a qubit junction with a shunt capacitor fabricated with a crystalline dielectric in a parallel-plate geometry. The small size of this element will give low radiation and surface loss, while the crystalline dielectric will have a low density of TLS. For this structure, a significant materials challenge will be epitaxial growth of an insulator on a metal, including good interface quality.

\section{$6.2 T_{2}$ : dephasing}

The quantity $T_{1}$ characterizes energy loss produced by transitions from the $|1\rangle$ to $|0\rangle$ state. An additional decoherence mechanism comes from dephasing, which can be thought of as noise in the relative phase between the two qubit states [29]. It is typically determined with a Ramsey fringe experiment, which measures a decoherence time called $T_{2}$. Energy loss also contributes to $T_{2}$ according to the relation $1 / T_{2}=1 / 2 T_{1}+1 / T_{\phi}$, where $1 / T_{\phi}$ is the dephasing rate, coming typically from low frequency noise, that does not cause a qubit transition. In this section, we are interested in the additional decoherence that comes from dephasing, as characterized by $T_{\phi}$.

Dephasing is one of the most critical decoherence mechanisms for superconducting qubits, and has mostly determined which qubit designs have been successful. Dephasing is equivalent to noise in the phase of the qubit state, which can be calculated from the time integral of fluctuations in the qubit energy, which in turn fluctuates from noise in qubit parameters. These parameters typically fluctuate with a strong low frequency component characterized by a $1 / f$ noise power spectrum.

The most important parameter fluctuations are charge, flux, and junction criticalcurrent. Charge qubits are most sensitive to charge noise, and the characteristic dephasing time for typical parameters give $T_{\phi} \sim 3 \mathrm{~ns}$. Flux qubits are similarly sensitive to flux noise, giving $T_{\phi} \sim 30 \mathrm{~ns}$. Because of these short coherence times, these devices are typically operated at a degeneracy point [52] where the device is not sensitive to charge or flux. Although this solution greatly slows down dephasing, the degeneracy point adds additional complications and constraints, especially when qubits need to be coupled.

The phase qubit does not have a degeneracy point, but neither is the dephasing rate as detrimental as for the other qubit designs. Present designs give $T_{\phi} \simeq 120-200 \mathrm{~ns}$, but with redesign may be improved to $\sim 1 \mu \mathrm{s}$. Control and coupling is much simpler without having to work around the physics of a degeneracy point, so phase qubits have been coupled together in more advanced experiments. The large capacitance of the phase qubit makes it insensitive to charge noise [29], a feature known long before it was incorporated in the transmon qubit [19]. The $1 / f$ flux noise spectrum has been directly measured using a phase qubit, showing it is the dominant dephasing mechanism [2].

Although the dephasing time is long enough for present qubit experiments, it clearly needs to be improved for advanced algorithms. Although the origin of flux noise has 
been an open question for over 20 years [13,55], I am optimistic that its magnitude can be reduced since new experiments have emerged that probe the flux noise mechanisms. In particular, a recent experiment [42] has measured the temperature dependence below $1 \mathrm{~K}$ of the flux through a SQUID. This data was simply explained with electron spins on the surface of the superconductor, with their measured density being consistent with the observed magnitude of $1 / f$ flux noise. Work is in progress to identify the microscopic mechanism that generates these surface spins $[9,20,40]$, from which new materials or surface treatments may emerge.

Since dephasing arises from low frequency noise, we have shown [29] that a simple classical calculation using noise theory can be used to understand the decoherence physics. In these calculations, the time dependence of decoherence was found to depend on the frequency scaling of the noise, with $1 / f$ noise producing dephasing that scales as time squared. The theory also describes how spin echo techniques may be used to significantly reduce the effects of dephasing [29].

\subsection{Measurement errors}

The qubit state is measured by pulsing current through the junction, which lowers the barrier of the cubic potential so that the $|1\rangle$ state may escape [4]. An amplification process takes place after escape, similar to an ionized atom in a electric field, so that the final classical state after tunneling is much different than when the state did not tunnel. This large change in state is then read out with a simple flux measurement using a SQUID.

The fidelity of this measurement is not perfect because the $|1\rangle$ state has a small probability not to tunnel, and the $|0\rangle$ state has small probability to tunnel. Fortunately, the ratio of the tunneling rates for these two states is large, about 200 for typical parameters, so that the two qubits states can be determined with a fidelity of about $96 \%$ when properly biased. In practice, the measurement fidelity is somewhat lower, due to relaxation of the qubit state during measurement. Presently, the dominant relaxation mechanism is transfer of energy to TLS, as predicted and observed in several experiments [4,24]. In addition, energy can be lost due to ordinary $T_{1}$ energy decay, with a probability of decay given the measurement time divided by $T_{1}$. Presently, measurement fidelities around $90 \%$ are routinely achieved with phase qubits, with the errors in fidelity well explained by these mechanisms [24].

The simplicity of our measurement enables us to make fast measurements on the nanosecond time scale. This allows us to precisely probe the qubit dynamics and simultaneously measure the states of two qubits [33]. This latter property is essential for demonstrating entangled states [48].

I note that qubit measurement has become slightly more difficult as $T_{1}$ has improved. As dissipation is lowered, the decay time of the final states are lengthened and the qubit state no longer tunnels into a continuum. Under this condition, we typically observed additional oscillation in the tunneling rate with qubit bias. When tunneling into a well with $n$ states, with typically $n \sim 300$, the decay time of the $n$-th state is roughly $T_{1} / n$. The measurement pulse should be longer than this time to allow this state to partially 
relax during measurement. This phenomenon has been theoretically analyzed in detail [21] and well describes experimental observations.

\subsection{Logic-gate errors}

Most discussions of coherence in qubits center around $T_{1}$ and $T_{2}$. However, these coherence times essentially describe decoherence of memory operations, and do not include possible error mechanisms when performing the actual quantum logic operations.

We have performed the first experiment in superconducting qubits to look at these types of gate errors [24]. In particular, we focused on logic errors from transitions to states out of the qubit subspace [50], specifically the $|2\rangle$ state, when performing single qubit operations. We have demonstrated that careful generation of Gaussian pulses can produce control signals with low power at the unwanted $|1\rangle$ to $|2\rangle$ transition. Based on a new measurement technique called a "Ramsey filter", we were able to measure and reduce these errors down to the $10^{-4}$ level [24].

\section{Tomography}

Our procedure for measuring qubits, by itself, does not completely determine the quantum state. A single measurement of a qubit state gives a (binary) output of 0 or 1 for the occupation of the qubit eigenstate $|1\rangle$. By identically preparing the state and repeating this measurement, an average occupation probability of the eigenstate can be found. This still does not completely determine the prepared state, since no phase information is revealed. In the Bloch sphere picture, we have only measured the Bloch vector projected along the $z$-direction.

Tomography allows the state to be fully determined by performing two additional sets of measurements along basis states that correspond to the $\mathrm{x}$ and $\mathrm{y}$ directions of the Bloch sphere. For superconducting qubits, this is accomplished by rotating the state to be measured by 90 degrees around the $y$ - or $x$-axis before measurement. From this projection (measurement) of the Bloch vector in the $x, y$, and $z$ directions, the Bloch vector can be reconstructed.

In the first tomography experiment for a superconducting qubit [49], the quantum state was initially rotated around an arbitrary axis on the equator and with a variable rotation angle. Although this procedure overconstrains the determination of the quantum state, it is a useful experimental check since errors in control or measurement can more easily be identified. In this experiment, state tomography for a decaying state was also demonstrated using the more efficient $x, y$, and $z$-axis projections.

Tomography can readily be generalized for multiple qubit states. It was first demonstrated for coupled superconducting qubits as a means to directly measure entanglement [48]. In this experiment, the entangled quantum state was measured along the 3 measurement axis for both qubits, giving 9 total measurements. From the three unique probabilities obtained for each of the 9 measurements, the density matrix was reconstructed using matrix inversion and a least squares fit. 
Quantum process tomography is used to characterize qubit logic operations from an arbitrary initial state to a final state. This is performed by measuring, via state tomography, the state transformation for a sampling of 4 initial states, typically chosen as $|0\rangle,|1\rangle(|0\rangle+|1\rangle) / \sqrt{2}$, and $(|0\rangle+\mathrm{i}|1\rangle) / \sqrt{2}$. The first process tomography experiment for superconducting qubits was performed for a memory operation between a phase qubit and a two-level state [34]. Process tomography showed a state transformation corresponding to a unity operation, as desired for memory, with a fidelity of $79 \%$.

\section{Qubit fabrication}

We have developed a multi-layer fabrication process for qubits that enables complex designs with wiring crossovers. Although crossovers are complicated to fabricate, they are essential for good microwave performance since they ensure good connection between all ground electrodes. In order to have a cleaner and more reliable process, we have avoided as much as possible the deposition of materials through shadow mask evaporation, instead using blanket deposition and etching processes. In particular, our fabrication step for Josephson junctions completely avoids the use of organic materials during deposition, such as those commonly found in shadow masks made from e-beam resist or photoresist.

Our standard qubit process uses 7 mask levels. For faster processing, we pattern our devices using photolithography in a wafer stepper. Line widths and spacings of $1 \mu \mathrm{m}$ can be achieved, but we routinely use design rules of $2 \mu \mathrm{m}$ to make processing more reliable. Step-edge Josephson junctions are regularly fabricated with areas $\sim 1 \mu \mathrm{m}^{2}$; significantly smaller junctions are possible after further optimization of photolithography [31].

Aluminum has been chosen as the superconductor since many other research groups have demonstrated a long $T_{1}$ using $\mathrm{Al}$. We are beginning to incorporate in the base layer a Re superconductor, which is an interesting metal since it tends to not form a surface oxide and may have lower loss.

An overview of our standard qubit fabrication process is given here.

1. Sapphire $\left(\mathbf{A l}_{2} \mathbf{O}_{3}\right)$ substrate. Silicon wafers are not used to avoid substrate conductance induced from charge defects in the insulator layer.

2. Al deposition and etch for base wiring. The Al base wiring layer is sputter deposited, then patterned and etched in a reactive ion etcher (RIE) using $\mathrm{BCl}_{3} / \mathrm{Cl}_{2}$.

3. a-Si:H insulator deposition and via etch. Amorphous hydrogenated silicon is used as an insulator and deposited using PECVD. After patterning, it is plasma etched with $\mathrm{CF}_{4}$. After this step, a-Si:H covers most of the wafer, except for vias used for contacts to the base wiring.

4. Al wiring layer and etch. The surface of the wafer is lightly cleaned with an Ar ion mill, and then the Al wiring layer is sputter deposited. It is patterned and etched as for the base wiring. In this step, the wiring layer remains in an area where the Josephson junction will be made, and directly contacts the substrate. The Josephson junction must be grown on the wiring layer, not the base layer, in order to obtain high quality current-voltage characteristics. 
5. Junction deposition and etch. The Josephson junction is fabricated over the entire substrate by first lightly cleaning the exposed $\mathrm{Al}$ with an $\mathrm{Ar}$ ion mill, then growing the tunnel barrier with thermal oxidation using $\mathrm{O}_{2}$ gas, and finally by sputtering the $\mathrm{Al}$ counterelectrode. After patterning, the junction is etched with a $\mathrm{Ar} / \mathrm{Cl}_{2}$ plasma. Etching arises from Ar milling, with $\mathrm{Cl}_{2}$ being used to scavenge and carry away the milled Al. The etch proceeds slightly into the wiring layer, and must be precisely timed as to not overetch the underlying wiring.

6. Al wiring etch. The patterning of the $\mathrm{Al}$ wiring layer is now completed using a $\mathrm{BCl}_{3} / \mathrm{Cl}_{2}$ etch.

7. a-Si:H etch. The insulating layer has been used to protect the base wiring layer from being etched. It is now removed by a $\mathrm{CF}_{4}$ plasma etch. Although lithographic patterning is used for this step, it is not strictly necessary.

8. Shorting strap etch. Shorting straps are used to protect the Josephson junctions from damage by plasma processing. After patterning, the shorting straps are removed by a liquid (acid) etch.

For devices made with a Re base electrode, the fabrication process is identical except that $\mathrm{SF}_{6}$ is used for plasma etching. A AuCu resistor layer may be incorporated into this process in the step before the last etch of the shorting strap. For simplicity, we evaporate this alloy through a photoresist liftoff mask.

\section{Control electronics}

Precise electrical waveforms need to be synthesized for controlling the qubits. The general requirements for electronics are reviewed here, as well as how they have been implemented in a reasonably scalable (low cost) manner.

The general requirements begin by considering the qubit transition frequency, which is typically chosen to be in the $5-7 \mathrm{GHz}$ range. This frequency is low enough to simplify microwave design, and thermal fluctuations at dilution refrigerator temperatures $(20 \mathrm{mK})$ are negligible. Next, the phase qubit has a non-linearity, as defined as the difference in the $|1\rangle \leftrightarrow|0\rangle$ and $|2\rangle \leftrightarrow|1\rangle$ transition frequencies, that is typically about $200 \mathrm{MHz}$. This value implies that microwave control pulses need to vary over a characteristic time scale of about $5 \mathrm{~ns}[24,50]$. This time scale also ensures the validity of the rotating wave approximation, an assumption that is made for most control schemes.

Control pulses are also needed to vary the qubit frequency and to pulse the bias current near the critical current for the tunneling measurement. The qubit frequency is typically changed by $100-400 \mathrm{MHz}$ to move it into and out of resonance, effectively turning on and off the coupling mechanism. This change in frequency also changes the phase of a qubit, giving a $2 \pi$ rotation in a few nanoseconds. This time scale implies that pulses bringing qubits into and out of resonance should have timing adjustable at the $10-100$ ps scale. The pulse rise time also needs to be fast, of order $1 \mathrm{~ns}$, in order to perform fast control and measurement.

The above requirements can be met with relatively low cost components based on a fast 14-bit digital to analog (D/A) converter chip that operates at 1 giga-sample per second. By sending its output through an anti-aliasing filter with $\sim 200 \mathrm{MHz}$ bandwidth, pulses with few ns rise time can by synthesized with adjustable delay times well below 
100 ps. For microwaves, two of these converters can be connected to a microwave quadrature mixer to generate an output microwave waveform with arbitrary amplitude, frequency and phase. From the Nyquist theorem, this mixer can produce an output frequency varying between about $\pm 400 \mathrm{MHz}$ around the input carrier frequency.

Synthesized waveforms allow complex control sequences to be generated easily. As importantly, we also find that a $\mathrm{GHz} \mathrm{D} / \mathrm{A}$ enables imperfections in the electronics to be readily corrected. By calibrating the response of the various filters and mixers with a sampling oscilloscope, the imperfections can be removed using deconvolution techniques. Similarly, we can correct for all imperfections in the bias lines down to the qubit by using the tunneling of the qubit as a sampling oscilloscope in a pump-probe configuration.

Precise Gaussian-shaped microwave pulses are synthesized in order to produce a waveform that is both minimum in time and frequency, reducing errors due to the excitation of the $|2\rangle$ state. Slepian shaped pulses [46] have also been synthesized since they have minimum frequency tails like Gaussians, but have hard cutoffs in the time domain.

We have designed and developed custom electronics based on commercially available D/A converters in order to keep the cost of the control electronics to a few thousand dollars per channel. Each board has two D/A converters driven by one high-performance field programmable gate array (FPGA) chip, with an onboard phase-locked loop generating the $1 \mathrm{GHz}$ clock from a $10 \mathrm{MHz}$ reference input. An important design criteria for high speed converters is obtaining clock jitter in the few ps range. We thus use a low phase-noise master clock and clock distribution system.

The phase qubit also needs to be biased with a relatively high current compared to the control signals. This control is not possible with our high speed D/A converters because they have large $1 / f$ noise, typically with a crossover frequency in the $\mathrm{MHz}$ range. Instead, we use a low noise 16-bit D/A converter with a slow $(\sim 5 \mu \mathrm{s})$ update time, but having low $1 / f$ noise and good temperature stability. This converter is controlled by an external FPGA, with the clock being turned off during the qubit operation for low noise operation. With a resistor at $4 \mathrm{~K}$, this current bias is added to the high-speed current in a custom bias tee placed next to the qubit mount.

Our system allows complete software control of both the calibration of the electronics and the generation of the qubit sequences.

\section{Summary and acknowledgements}

In summary, the basic operations needed for quantum computation have been demonstrated in superconducting phase qubits. Although the coherence of phase qubits is somewhat less than for other types, the relative ease of coupling has enabled the demonstration of complex two-qubit experiments. Entangled quantum states and quantum logic operations have been measured precisely with tomographic techniques, and experiments using 20-30 control pulses have been performed with good fidelity. Although coherence needs to be improved in all superconducting qubits, we now understand many of the decoherence mechanisms so that dramatic improvements in 
materials and device design are well within reach. A detailed description has been given for our fabrication process and control electronics, which we believe is directly scalable. Experiments are now underway to demonstrate more complex multi-qubit algorithms.

Devices were made at the UCSB and Cornell Nanofabrication Facility, a part of the NSF-funded National Nanotechnology Infrastructure Network. This work was supported by IARPA under grant W911NF-04-1-0204 and by the NSF under grant CCF-0507227.

Open Access This article is distributed under the terms of the Creative Commons Attribution Noncommercial License which permits any noncommercial use, distribution, and reproduction in any medium, provided the original author(s) and source are credited.

\section{References}

1. Berkley, A.J., Xu, H., Ramos, R.C., Gubrud, M.A., Strauch, F.W., Johnson, P.R., Anderson, J.R., Dragt, A.J., Lobb, C.J., Wellstood, F.C.: Entangled macroscopic quantum states in two superconducting qubits. Science 300, 1548-1550 (2003)

2. Bialczak, R.C., McDermott, R., Ansmann, M., Hofheinz, M., Katz, N., Lucero, E., Neeley, M., O'Connell, A., Wang, H., Cleland, A.N., Martinis, J.M.: 1/f flux noise in Josephson phase qubits. Phys. Rev. Lett. 99, 187006 (2007)

3. Clarke, J., Cleland, A.N., Devoret, M.H., Martinis, J.M.: Quantum-mechanics of a macroscopic variable - the phase difference of a Josephson junction. Science 239, 992-997 (1988)

4. Cooper, K.B., Steffen, M., McDermott, R., Simmonds, R., Oh, S., Hite, D., Pappas, D., Martinis, J.: Observation of quantum oscillations between a Josephson phase qubit and a microscopic resonator using fast readout. Phys. Rev. Lett. 93, 180401 (2004)

5. Devoret, M.H., Esteve, D., Martinis, J.M., Urbina, C.: Effect of an adjustable admittance on the macroscopic energy levels of a current biased Josephson junction. Phys. Scr. T25, 118-121 (1989)

6. Devoret, M.H., Martinis, J.M.: Implementing qubits with superconducting integrated circuits. Quantum Inf. Process. 3, 163-203 (2004)

7. Devoret, M.H., Martinis, J.M., Clarke, J.: Measurements of macroscopic quantum tunneling out of the zero-voltage state of a current-biased Josephson junction. Phys. Rev. Lett. 55, 1908-1911 (1985)

8. Esteve, D., Devoret, M.H., Martinis, J.M.: Effect of an arbitrary dissipative circuit on the quantum energy-levels and tunneling of a Josephson junction. Phys. Rev. B 34, 158-163 (1986)

9. Faoro, L., Ioffe, L.B.: Microscopic origin of low-frequency flux noise in Josephson circuits. Phys Rev. Lett. 100, 227005 (2008)

10. Gao, J., Daal, M., Martinis, J.M., Mazin, B.A., Day, P.K., Leduc, H.G., Vayonakis, A., Sadoulet, B., Zmuidzinas, J.: A semi-empirical model for two-level system noise in superconducting microresonators. Appl. Phys. Lett. 92, 212504 (2008)

11. Gao, J., Daal, M., Vayonakis, A., Kumar, S., Zmuidzinas, J., Sadoulet, B., Mazin, B.A., Day, P.K., Leduc, H.G.: Experimental evidence for a surface distribution of two-level systems in superconducting lithographed microwave resonators. Appl. Phys. Lett. 92, 152505 (2008)

12. Guerlin, C., Bernu, J., Deléglise, S., Sayrin, C., Gleyzes, S., Kuhr, S., Brune, M., Raimond, J.M., Haroche, S.: Progressive field-state collapse and quantum non-demolition photon counting. Nature 448, 889-893 (2007)

13. Harlingen, D.J.V., Robertson, T.L., Plourde, B.L.T., Reichardt, P.A., Crane, T.A., Clarke, J.: Decoherence in Josephson-junction qubits due to critical-current fluctuations. Phys. Rev. B 70, 064517 (2004)

14. Hofheinz, M., Wang, H., Ansmann, M., Bialczak, R.C., Lucero, E., Neeley, M., O’Connell, A.D., Sank, D., Wenner, J., Martinis, J.M., Cleland, A.N.: Synthesising arbitrary quantum states in a superconducting resonator. Nature (2009) (submitted)

15. Hofheinz, M., Weig, E.M., Ansmann, M., Bialczak, R.C., Lucero, E., Neeley, M., O’Connell, A.D., Wang, H., Martinis, J.M., Cleland, A.N.: Generation of fock states in a superconducting quantum circuit. Nature 454, 310-314 (2008) 
16. Ioffe, L.B., Geshkenbein, V.B., Helm, C., Blatter, G.: Decoherence in superconducting quantum bits by phonon radiation. Phys. Rev. Lett. 93, 057001 (2004)

17. Katz, N., Ansmann, M., Bialczak, R.C., Lucero, E., McDermott, R., Neeley, M., Steffen, M., Weig, E.M., Cleland, A.N., Martinis, J.M., Korotkov, A.N.: Coherent state evolution in a superconducting qubit from partial-collapse measurement. Science 312, 1498-1500 (2006)

18. Katz, N., Neeley, M., Ansmann, M., Bialczak, R.C., Hofheinz, M., Lucero, E., O’Connell, A., Wang, H., Cleland, A.N., Martinis, J.M., Korotkov, A.N.: Uncollapsing of a quantum state in a superconducting phase qubit. Phys. Rev. Lett. 101, 200401 (2008)

19. Koch, J., Yu, T.M., Gambetta, J., Houck, A.A., Schuster, D.I., Majer, J., Blais, A., Devoret, M.H., Girvin, S.M., Schoelkopf, R.J.: Charge-insensitive qubit design derived from the cooper pair box. Phys. Rev. A 76, 042319 (2007)

20. Koch, R.H., DiVincenzo, D.P., Clarke, J.: Model for 1/f flux noise in squids and qubits. Phys. Rev. Lett. 98, 267003 (2007)

21. Kofman, A., Zhang, Q., Martinis, J., Korotkov, A.: Theoretical analysis of measurement crosstalk for coupled Josephson phase qubits. Phys Rev. B 75, 014524 (2007)

22. Lang, K., Nam, S., Aumentado, J., Urbina, C., Martinis, J.M.: Banishing quasiparticles from Josephson-junction qubits: why and how to do it. IEEE Trans. Appl. Supercond. 13, 989 (2003)

23. Leggett, A.J.: Macroscopic quantum systems and the quantum theory of measurement. Prog. Theor. Phys. 69, 80 (1980)

24. Lucero, E., Hofheinz, M., Ansmann, M., Bialczak, R.C., Katz, N., Neeley, M., O’Connell, A., Wang, H., Cleland, A.N., Martinis, J.M.: High-fidelity gates in a Josephson qubit. Phys. Rev. Lett. 100, 247001 (2008)

25. Majer, J., Chow, J.M., Gambetta, J.M., Koch, J., Johnson, B.R., Schreier, J.A., Frunzio, L., Schuster, D.I., Houck, A.A., Wallraff, A., Blais, A., Devoret, M.H., Girvin, S.M., Schoelkopf, R.J.: Coupling superconducting qubits via a cavity bus. Nature 449, 443 (2007)

26. Martin, I., Bulaevskii, L., Shnirman, A.: Tunneling spectroscopy of two-level systems inside a Josephson junction. Phys. Rev. Lett. 95, 127002 (2005)

27. Martinis, J.M., Cooper, K., McDermott, R., Steffen, M., Ansmann, M., Osborn, K., Cicak, K., Oh, S., Pappas, D., Simmonds, R., Yu, C.C.: Decoherence in Josephson qubits from dielectric loss. Phys. Rev. Lett. 95, 210503 (2005)

28. Martinis, J.M., Devoret, M.H., Clarke, J.: Energy-level quantization in the zero-voltage state of a current-biased Josephson junction. Phys. Rev. Lett. 55, 1543-1546 (1985)

29. Martinis, J.M., Nam, S., Aumentado, J., Lang, K., Urbina, C.: Decoherence of a superconducting qubit from bias noise. Phys. Rev. B 67, 94510 (2003)

30. Martinis, J.M., Nam, S., Aumentado, J., Urbina, C.: Rabi oscillations in a large Josephson-junction qubit. Phys. Rev. Lett. 89, 117901 (2002)

31. Martinis, J.M., Ono, R.H.: Fabrication of ultrasmall nb-alox-nb Josephson tunnel-junctions. Appl. Phys. Lett. 57, 629-631 (1990)

32. McDaniel H.Y.: Vortex dissipation in type i superconducting films. Undergraduate thesis, University of California, Santa Barbara (2006)

33. McDermott, R., Simmonds, R., Steffen, M., Cooper, K., Cicak, K., Osborn, K., Oh, S., Pappas, D., Martinis, J.: Simultaneous state measurement of coupled Josephson phase qubits. Science 307, 12991302 (2005)

34. Neeley, M., Ansmann, M., Bialczak, R.C., Hofheinz, M., Katz, N., Lucero, E., O’Connell, A., Wang, H., Cleland, A.N., Martinis, J.M.: Process tomography of quantum memory in a Josephson-phase qubit coupled to a two-level state. Nat. Phys. 4, 523-526 (2008)

35. Neeley, M., Ansmann, M., Bialczak, R.C., Hofheinz, M., Katz, N., Lucero, E., O’Connell, A., Wang, H., Cleland, A.N., Martinis, J.M.: Transformed dissipation in superconducting quantum circuits. Phys. Rev. B 77, 180508 (2008)

36. O’Connell, A.D., Ansmann, M., Bialczak, R.C., Hofheinz, M., Katz, N., Lucero, E., McKenney, C., Neeley, M., Wang, H., Weig, E.M., Cleland, A.N., Martinis, J.M.: Microwave dielectric loss at single photon energies and millikelvin temperatures. Appl. Phys. Lett. 92, 112903 (2008)

37. Osbourne, K., Martinis, J.M.: Superconducting qubits and the physics of Josephson junctions. In: Les Houches Conference Proceedings (2003)

38. Pohl, R.O., Liu, X., Thompson, E.: Low-temperature thermal conductivity and acoustic attenuation in amorphous solids. Rev. Mod. Phys. 74, 991-1013 (2002) 
39. Ramos, R.C., Gubrud, M.A., Berkley, A.J., Anderson, J.R., Lobb, C.J., Wellstood, F.C.: Design for effective thermalization of junctions for quantum coherence. IEEE Trans. Appl. Supercond. 11, 9981001 (2001)

40. Rogachev, A., Wei, T.C., Pekker, D., Bollinger, A.T., Goldbart, P.M., Bezryadin, A.: Magnetic-field enhancement of superconductivity in ultranarrow wires. Phys. Rev. Lett. 97, 137001 (2006)

41. Schickfus, M.v., Hunklinger, S.: The dielectric coupling of low-energy excitations in vitreous silica to electromagnetic waves. J. Phys. C9, L439 (1976)

42. Sendelbach, S., Hover, D., Kittel, A., Mück, M., Martinis, J.M., McDermott, R.: Magnetism in squids at millikelvin temperatures. Phys. Rev. Lett. 100, 227006 (2008)

43. Shnirman, A., Schn, G., Martin, I., Makhlin, Y.: Low- and high-frequency noise from coherent twolevel systems. Phys. Rev. Lett. 94, 127002 (2005)

44. Sillanpa, M.A., Park, J.I., Simmonds, R.W.: Coherent quantum state storage and transfer between two phase qubits via a resonant cavity. Nature 438, 442 (2007)

45. Simmonds, R., Lang, K., Hite, D., Pappas, D., Martinis, J.: Decoherence in Josephson phase qubits from junction resonators. Phys. Rev. Lett. 93, 077003 (2004)

46. Slepian, D.: Prolate spheroidal wave functions, fourier analysis, and uncertainty. v. the discrete case. Bell Syst. Tech. J. 57, 1371 (1978)

47. Stan, G., Field, S., Martinis, J.: Critical field for complete vortex expulsion from narrow superconducting strips. Phys. Rev. Lett. 92, 097003 (2004)

48. Steffen, M., Ansmann, M., Bialczak, R.C., Katz, N., Lucero, E., McDermott, R., Neeley, M., Weig, E.M., Cleland, A.N., Martinis, J.M.: Measurement of the entanglement of two superconducting qubits via state tomography. Science 313, 1423-1425 (2006)

49. Steffen, M., Ansmann, M., McDermott, R., Katz, N., Bialczak, R.C., Lucero, E., Neeley, M., Weig, E., Cleland, A., Martinis, J.M.: State tomography of capacitively shunted phase qubits with high fidelity. Phys. Rev. Lett. 97, 050502 (2006)

50. Steffen, M., Martinis, J., Chuang, I.: Accurate control of Josephson phase qubits. Phys. Rev. B 68, 224518 (2003)

51. Urbina, C., Esteve, D., Martinis, J.M., Turlot, E., Devoret, M.H., Grabert, H., Linkwitz, S.: Measurement of the latency time of macroscopic quantum tunneling. Physica B 169, 1-4 (1991)

52. Vion, D., Aassime, A., Cottet, A., Joyez, P., Pothier, H., Urbina, C., Esteve, D., Devoret, M.H.: Manipulating the quantum state of an electrical circuit. Science 296, 886 (2002)

53. Wallraff, A., Schuster, D.I., Blais, A., Frunzio, L., Huang, R.S., Majer, J., Kumar, S., Girvin, S.M., Schoelkopf, R.J.: Strong coupling of a single photon to a superconducting qubit using circuit quantum electrodynamics. Nature 431, 162-167 (2004)

54. Wang, H., Hofheinz, M., Ansmann, M., Bialczak, R.C., Lucero, E., Neeley, M., O’Connell, A.D., Sank, D., Wenner, J., Cleland, A.N., Martinis, J.M.: Measurement of the decay of Fock state in a superconducting quantum circuit. Phys. Rev. Lett. 101, 240401 (2008)

55. Wellstood, F.C., Urbina, C., Clarke, J.: Low-frequency noise in dc superconducting quantum interference devices below 1 k. Appl. Phys. Lett. 50, 772 (1987) 